\title{
Modelado y Simulación de un Seccionador Tensión- Tiempo para Protección Inteligente con Inclusión de Generación Distribuida de Electricidad
}

\author{
Jorge C. Vaschetti ${ }^{(1)}$, Juan C. Gomez ${ }^{(2)}$ y Juan C. Amatti ${ }^{(2)}$ \\ (1) GECaP, Facultad Regional Córdoba, Universidad Tecnológica Nacional, Maestro M. Lopez \\ esq. Cruz Roja Argentina, Ciudad Universitaria, X5016ZAA Córdoba-Argentina. \\ (e-mail: jvaschetti@scdt.frc.utn.edu.ar). \\ (2) IPSEP, Facultad de Ingeniería, Universidad Nacional de Río Cuarto, X5804BYA Río Cuarto, \\ Córdoba-Argentina.
}

Recibido Jul. 21, 2011; Aceptado Oct. 03, 2011; Versión Final recibida Oct. 15, 2011

\begin{abstract}
Resumen
Se presenta el modelado y simulación de un dispositivo que forma parte de un esquema de protección inteligente. Se propone un seccionador tensión-tiempo como base del sistema de protecciones aplicado a un distribuidor urbano-rural de electricidad. Se muestra el impacto de la generación distribuida en el esquema implementado y la utilización de un software de propósito general como herramienta de simulación. Este software permite implementar la máquina de estado finito que modela el seccionador propuesto, base del esquema de protecciones. Un resultado importante de este trabajo es que la utilización del dispositivo propuesto mantiene la coordinación aún frente a la inclusión de la generación distribuida. Se establece también con claridad la máxima cantidad de seccionadores que pueden ser interconectados en serie.
\end{abstract}

Palabras clave: sistema de protección inteligente, electricidad, generación distribuida, seccionador tensión-tiempo

\section{Modeling and Simulation of an Voltage-Time Sectionalizer for Intelligent Protection including Distributed Generation of Electricity}

\begin{abstract}
The modeling and simulation of a device that is part of an intelligent protection scheme. A voltage-time sectionalizer is proposed, as the basic unit of the system applied to an urban-rural distributor of electricity. The impact of distributed generation in the implemented scheme using a general purpose software simulation tool is demonstrated. This software allows implementing the finite state machine that models the proposed section of the protection scheme. An important result of this study is that the use of the proposed device maintains the coordination even with the inclusion of distributed generation. It is also clearly established the maximum number of sectionalizers that can be interconnected in series.
\end{abstract}

Keywords: intelligent protection system, electricity, distributed generation, voltage-time sectionalizer 


\section{INTRODUCCIÓN}

Tradicionalmente los sistemas de distribución fueron diseñados para llevar la energía eléctrica a los usuarios finales en forma radial. El sistema de distribución del futuro es un concepto nuevo que plantea como objetivos la flexibilidad y la controlabilidad como herramientas para facilitar el intercambio de energía y de información entre los participantes y los componentes con el objeto de mejorar las prestaciones (Sutherland et al., 2006).

Para cumplir con los objetivos planteados en el párrafo anterior se necesita de una serie de dispositivos como trasformadores universales inteligentes, capacitores conmutados, supresores de huecos de tensión, reguladores de voltaje, generación distribuída multifunción, seccionadores inteligentes, entre otros dispositivos; esquemas tales como controles basados en comunicaciones y aplicaciones por internet, alimentadores duales reconfigurados automáticamente son también utilizados (Sutherland et al., 2006; Kazemi et al., 2009).

Las protecciones inteligentes mejoran notablemente las prestaciones de los sistemas de distribución, esto se puede valorar calculando los índices de frecuencia de interrupción permanente y momentánea (SAIFI System Average Interruption Frequency Index, MAIFI Momentary Average Interruption Frequency Index, menos de cinco minutos) entre otros, en los que claramente se muestra la mejora obtenida como consecuencia de la utilización de dispositivos inteligentes direccionales (Kazemi et al., 2009).

La influencia de la inclusión de generación distribuida por si misma y de los sistemas de distribución inteligentes en los esquemas de protecciones tradicionales, hacen que deba estudiarse con cuidado la preservación de la coordinación de los dispositivos tradicionales, reconectador - seccionador - fusible; se deben considerar nuevos equipos que posibiliten los esquemas inteligentes (Walling et al., 2007).

En el presente trabajo se estudia un dispositivo el cual es propuesto para los sistemas de distribución inteligente, que si bien hace tiempo que se conoce su difusión no ha sido masiva hasta el momento. Un aspecto importante que se menciona de él es la posibilidad ilimitada de su conexión serie no mostrándose en los estudios y aplicaciones más de dos (Walling et al., 2007; Domo, 1989; Kazemi et al., 2009; Sutherland et al., 2006; COOPER). Otra característica del seccionador tensión tiempo es que la coordinación no depende de la corriente de cortocircuito, por lo tanto, una vez sincronizado con el re-conectador no es necesario su reajuste lo que hace su utilización muy atractiva para aplicaciones con generación distribuída (Domo, 1989).

Para realizar el estudio propuesto se recurre a un software de propósito general con el cual se sintetiza la lógica de operación de los dos dispositivos fundamentales que se utilizan, el reconectador y el seccionador tensión-tiempo, se modela y simula un cadena serie, se muestra la condición de sincronización, los límites de la cadena y se comprueba el mantenimiento de la coordinación.

En la primera parte se presenta un esquema tradicional de protecciones con la generación distribuída incluida mostrando los efectos de su inclusión, seguidamente se efectúa un análisis sobre las ventajas de utilizar un sistema de protección inteligente para luego abordar directamente el seccionador tensión-tiempo finalizando con la exposición de los resultados y las conclusiones.

\section{PROTECCIÓN CLÁSICA CON INCLUSIÓN DE LA GENERACIÓN DISTRIBUIDA}

Se trata de un sistema de distribución radial en media tensión con dos distribuidores urbanos que en sus colas de líneas alimentan zonas rurales, mientras que en su zona urbana provee energía a distintas industrias y hogares, el mismo se presenta en la Fig. 1. La generación distribuída está a cargo de cooperativas regionales (Gen2, Gen3 y Gen4). 


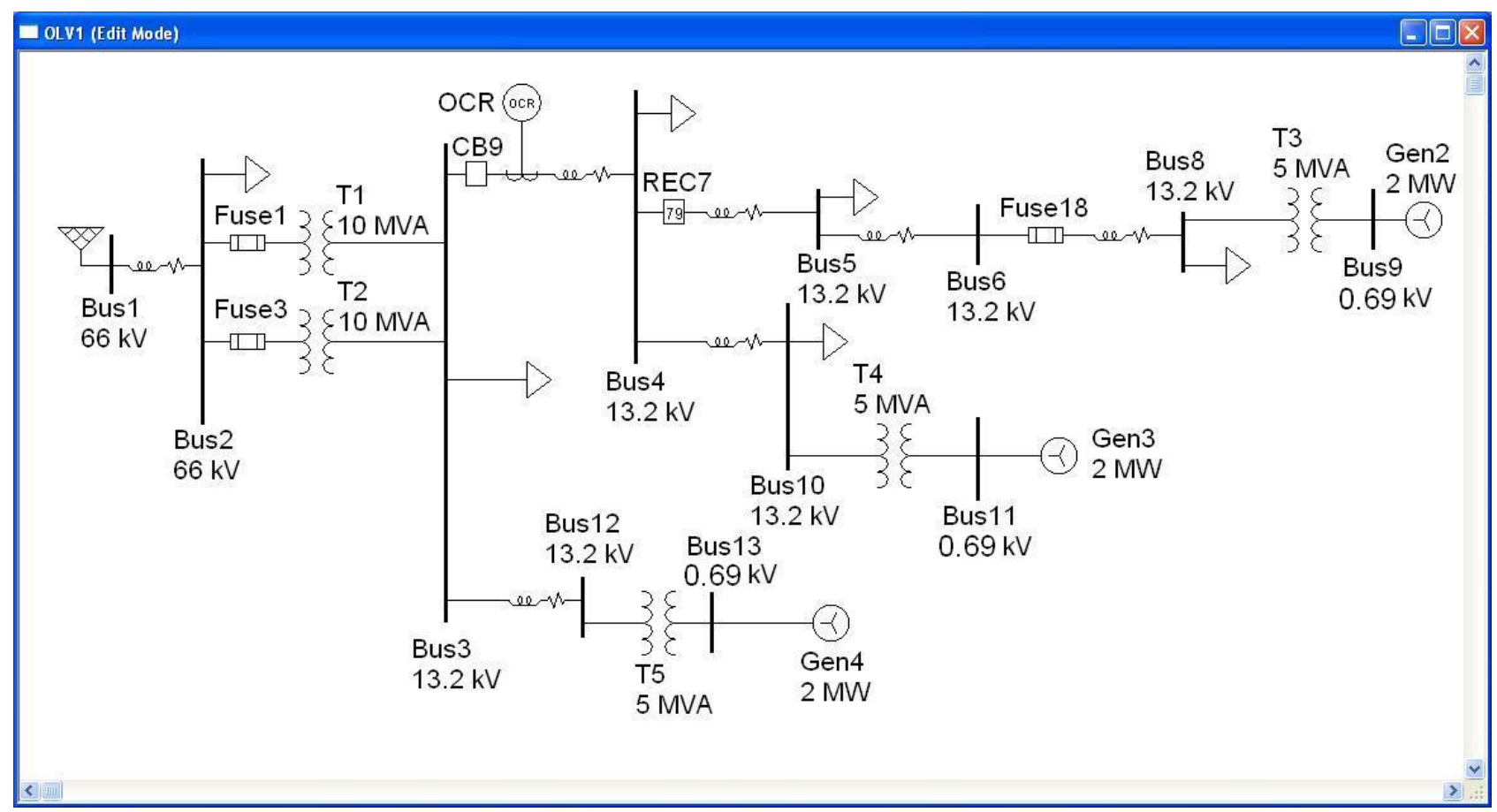

Fig. 1. Sistema de distribución radial urbano-rural bajo estudio con generación distribuida incorporada.

Dada la estructura del sistema de distribución, se propone proteger los ramales rurales con fusibles (Fuse18), re-conectadores (REC7) y relé de sobre corriente en el distribuidor principal (OCR CB9), protegiéndose a cada transformador con fusible del lado de alta (Fuse1, Fuse3), en la Fig. 1 se presenta el esquema bajo análisis solamente para todo lo que implique el ramal superior (IEEE C 37.230, 2008). El esquema propuesto se simula sin generación distribuida utilizando el software ETAP 6.0 comprobándose la coordinación de los distintos dispositivos.

Para analizar la influencia de la generación distribuida en el sistema implementado se deben tener en cuenta los siguientes aspectos (Dysko et al., 2007):

a) Cada caso debe ser analizado en forma particular, no existiendo una ley general.

b) La incidencia de la generación distribuida en el sistema de protecciones depende del lugar de inserción y de la potencia instalada.

c) Las protecciones para generación distribuida exigidas por el ente regulador ante perturbaciones, permitirían mantener el esquema original del sistema de distribución, siempre y cuando las primeras actúen en tiempo y forma antes que las segundas.

Se repite nuevamente la simulación con inclusión de la generación distribuida aplicando un cortocircuito trifásico en la barra 8 Fig. 1 (Bus 8), se puede visualizar el riesgo de una descoordinación, Fig. 2, ya que el fusible (Fuse18 33,3 ms) puede operar en el ciclo rápido del reconectador el cual está pensado para permitir el despeje de fallas transitorias (REC7 37,9 ms), por lo tanto, se hace necesario reajustar esta coordinación.

La Fig. 3 muestra el diagrama de tiempos de la secuencia de operaciones, en ella se aprecia la ajustada coordinación entre el fusible y el re-conectador, habría que reajustar el fusible, esto muestra que lo que estaba coordinado sin generación distribuida puede no estarlo con su inclusión. 


\section{SISTEMA DE PROTECCIÓN INTELIGENTE}

La inserción de generación distribuida puede ocasionar la descoordinación entre los dispositivos, sería importante contar con algún esquema superador de las protecciones tradicionales que permita operar con una mayor confiabilidad.

En las Fig. 4 y Fig. 5 se presenta un estudio en el que claramente se muestra la mejora obtenida en los índices SAIFI y MAIFI como consecuencia de la utilización de dispositivos inteligentes direccionales (Kazemi et al., 2009).

\begin{tabular}{|c|c|c|c|c|c|c|}
\hline \multicolumn{7}{|c|}{ Sequence-of-0peration Events - Output Report: TPFinal } \\
\hline \multicolumn{7}{|c|}{ 3-Phase (Symmetrical) fault on bus: Barra 8} \\
\hline \multicolumn{4}{|c|}{ Data Rev.: Base } & \multicolumn{2}{|c|}{ Config: Normal Date: $10-17-2010$} & \\
\hline Time (ms) & ID & If $(\mathrm{kA})$ & $\mathrm{T} 1(\mathrm{~ms})$ & $\mathrm{T} 2$ (ms) & Condition & \\
\hline 37,9 & REC7 & 1,634 & 9,9 & 37,9 & 1st Operation - Phase - Fast - TOC & \\
\hline 74,9 & Fuse18 & 1,634 & 33,3 & 74,9 & & \\
\hline 538 & REC7 & & 500 & & 1st Recloser & \\
\hline 576 & REC7 & 1,634 & 9,9 & 37,9 & 2nd Operation - Phase - Fast - TOC & \\
\hline 1076 & $\mathrm{REC7}$ & & 500 & & 2nd Recloser & \\
\hline 1999 & REC7 & 1,634 & 895 & 923 & 3rd Operation - Phase - Delay - TOC & \\
\hline 2499 & REC7 & & 500 & & 3rd Recloser & \\
\hline 3422 & REC7 & 1,634 & 895 & 923 & 4th Operation - Lockout - Phase - Delay - TOC & \\
\hline 3676 & Relay9 & 1,321 & 3676 & & Phase - oc1 - 51 & \\
\hline 3759 & $\mathrm{CBO}$ & & 83,3 & & Tripped by Relay9 Phase - OC1 - 51 & \\
\hline
\end{tabular}

Fig. 2. Secuencia de operaciones falla trifásica barra 8 con generación distribuida incorporada.

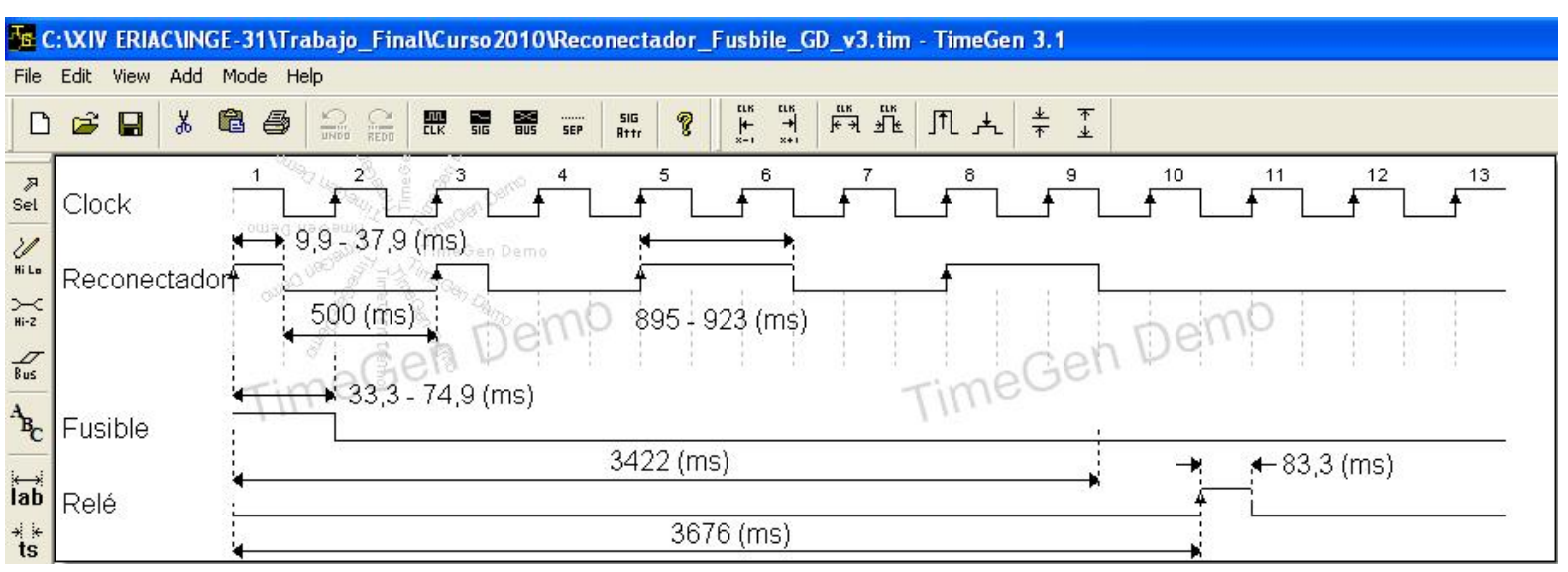

Fig. 3. Diagrama de tiempos de la secuencia de operaciones de la Fig. 2.

Se intercala un dispositivo que funciona en forma coordinada con el re-conectador ubicado en la cabecera del alimentador, Fig. 4, se plantean distintos casos para distintos tipos de dispositivos intermedios y se evalúan los índices para cada uno (Fahey et al. 2008). En la Fig. 5 podemos apreciar una configuración en la que se operan dos alimentadores con dispositivos inteligentes direccionales, en este caso es clara la mejora en los índices, es importante hacer notar que para el caso de una falla permanente el tiempo de reparación es casi independiente del dispositivo usado.

Una conclusión lógica que se puede extraer del estudio anterior es que la utilización de componentes con capacidad de re-cierre mejora sustancialmente los índices, cosa que por otra parte no debería llamar la atención dada la capacidad de despejar fallas transitorias que tienen éstos, si a ello se le agrega la independencia en el tiempo de reparación para una falla permanente, se vuelve atractivo su empleo. 


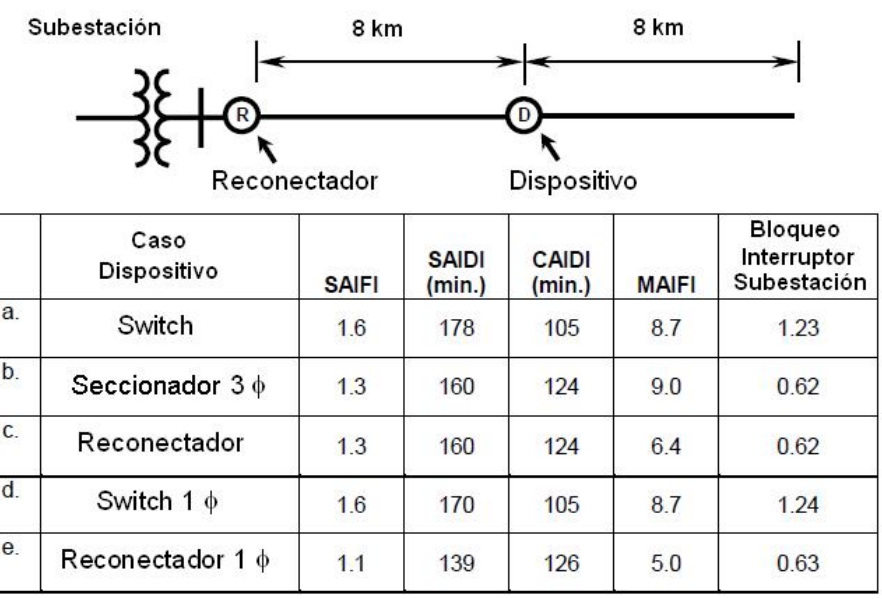

Fig. 4. Localización de dispositivos de protección intermedios en el alimentador.

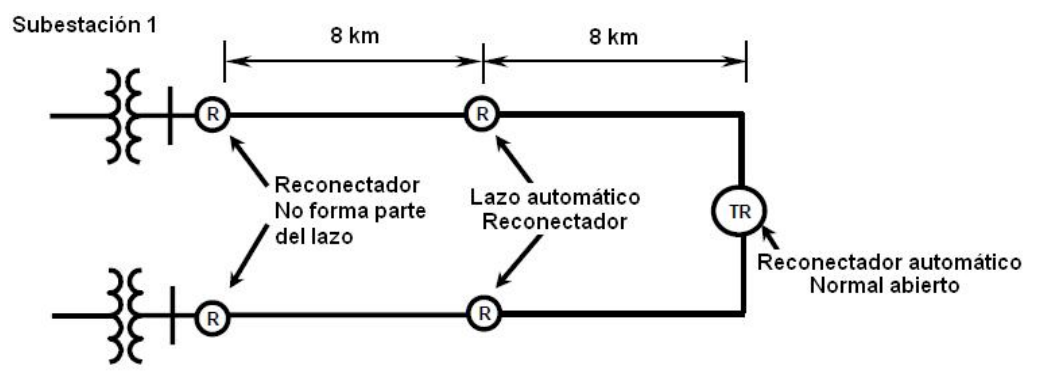

Subestación 2

\begin{tabular}{|c|c|c|c|c|c|c|}
\hline & Caso & SAIFI & $\begin{array}{c}\text { SAIDI } \\
\text { (min.) }\end{array}$ & $\begin{array}{c}\text { CAIDI } \\
\text { (min.) }\end{array}$ & MAIFI & $\begin{array}{c}\text { Interruptor } \\
\text { Subestación }\end{array}$ \\
\hline a. & Reconectador 3 $\$$ & 1.0 & 122 & 124 & 6.7 & 0.62 \\
\hline b. & Reconectador 1 $\phi$ & 0.8 & 99 & 126 & 5.3 & 0.63 \\
\hline
\end{tabular}

Fig. 5. Sistema de doble alimentador operado automáticamente.

\section{MODELADO Y SIMULACIÓN DEL SECCIONADOR TENSION - TIEMPO}

\section{Características, principio de funcionamiento}

Los seccionadores tensión - tiempo presentan una fácil coordinación con el re-conectador de alimentación, no perdiéndose la misma una vez establecida ésta; no se corre el riesgo ante una reposición de servicio de que por reemplazo inadecuado, caso fusible, se pierda; otra gran ventaja de estos dispositivos es que no hay que preocuparse por la corriente de inserción de los transformadores (inrush current) dado que actúan por una combinación tensión - tiempo (Domo, 1989).

Representan también una solución para la generación distribuída dado que no responden a sobre corrientes, la coordinación con el re-conectador no se ve afectada por la presencia de ésta como es el caso del fusible o del seccionador operado por conteo de operaciones del re-conectador. Los que operan por tensión - tiempo permiten implementaciones inteligentes mediante controles computarizados. Si bien en distintas publicaciones se menciona la posibilidad de conectar en serie con el re-conectador respectivo una cantidad ilimitada de éstos, no se muestra en ninguna de ellas esquema alguno, se debe tener en cuenta que los seccionadores tradicionales pueden conectarse en serie un máximo de dos (Domo, 1989).

Se presenta a continuación un modelado y simulación que permite demostrar la posibilidad de conexión ilimitada. Para entender el modelo lógico dinámico de este seccionador se debe tener en 
cuenta el modo de operación del mismo que cuenta con tres temporizadores y un detector de voltaje, los cuales operan de la siguiente manera (COOPER):

1) Umbral de pérdida de tensión (Voltage Loss Threshold), permite seleccionar el umbral de detección para falta de voltaje (Voltaje loss) a bornes de entrada.

2) Tiempo de Apertura (Open Time), retardo de tiempo a la apertura ante la detección de falta de voltaje a bornes de entrada.

3) Tiempo de Cierre (Close Time), retardo de tiempo al cierre ante la detección de reposición del voltaje a bornes de entrada.

4) Tiempo de Inicialización (Reset Time), tiempo de accionamiento para el bloqueo ante la reposición del voltaje a bornes de entrada.

Si el dispositivo detecta falta de voltaje (Voltage Loss), espera un tiempo (Open time) para efectuar la apertura de contactos, una vez que haya seccionado y ante la reposición del voltaje de entrada espera un tiempo para el re-cierre (Close time), si producido éste se vuelve a perder el voltaje durante el tiempo de inicialización (Reset time) el seccionador abre y permanece bloqueado hasta su reposición manual.

Máquina de estado finito para el seccionador tensión - tiempo

Para simular una cadena re-conectador / seccionador se recurre al paquete de simulación compuesto por Matlab/Simulink ${ }^{\circledR}$, en la Fig. 6 se aprecia la máquina de estado finito que modela el comportamiento del seccionador tensión-tiempo.

El autómata presenta tres estados, seccionador cerrado (On), seccionador abierto (Off) y seccionador bloqueado (Block), la transición entre un estado y otro depende de los siguiente eventos:

1) On to Off, transición que depende del evento asíncrono entrada baja (inlow_event), el cual depende a su vez del dispositivo conectado inmediatamente aguas arriba, una transición alto-bajo del dispositivo superior dispara una transición del seccionador bajo consideración, también este evento depende del temporizador Open Time.

2) Off to On, transición que depende del evento temporal Close Time y de la cantidad de re-cierres que haya efectuado el seccionador.

3) On to Block, transición que opera según el evento temporal sincrónico reloj (clock_event) el cual depende del temporizador Reset Time y de la cantidad de re-cierres que haya efectuado el seccionador.

Además de los estados y transiciones se ejecutan piezas de código, función encendido (FOn) y función apagado (FOff), en las cuales se monitorean las entradas, se definen las salidas y se disminuyen y/o incrementan los temporizadores asociados.

Para el re-conectador se ha desarrollado una máquina de estado finito similar a la del seccionador, como se desprende de la propia lógica de operación del re-conectador, esta máquina resulta más compleja que la anterior y se hacen necesarias más funciones para representar la dinámica de la misma.

\section{Sistema de protección re-conectador - seccionador tensión - tiempo}

En la Fig. 7 se presenta un diagrama de simulación compuesto por un re-conectador y un seccionador, no existen inconvenientes en agregar más seccionadores a la cadena, solo se ha considerado uno a los fines de clarificar la figura. 
Es posible simular una falla en cualquier lugar de la cadena, en este caso es a partir del seccionador 3, cumplido el ciclo debe quedar abierto y despejar la falla permanente.

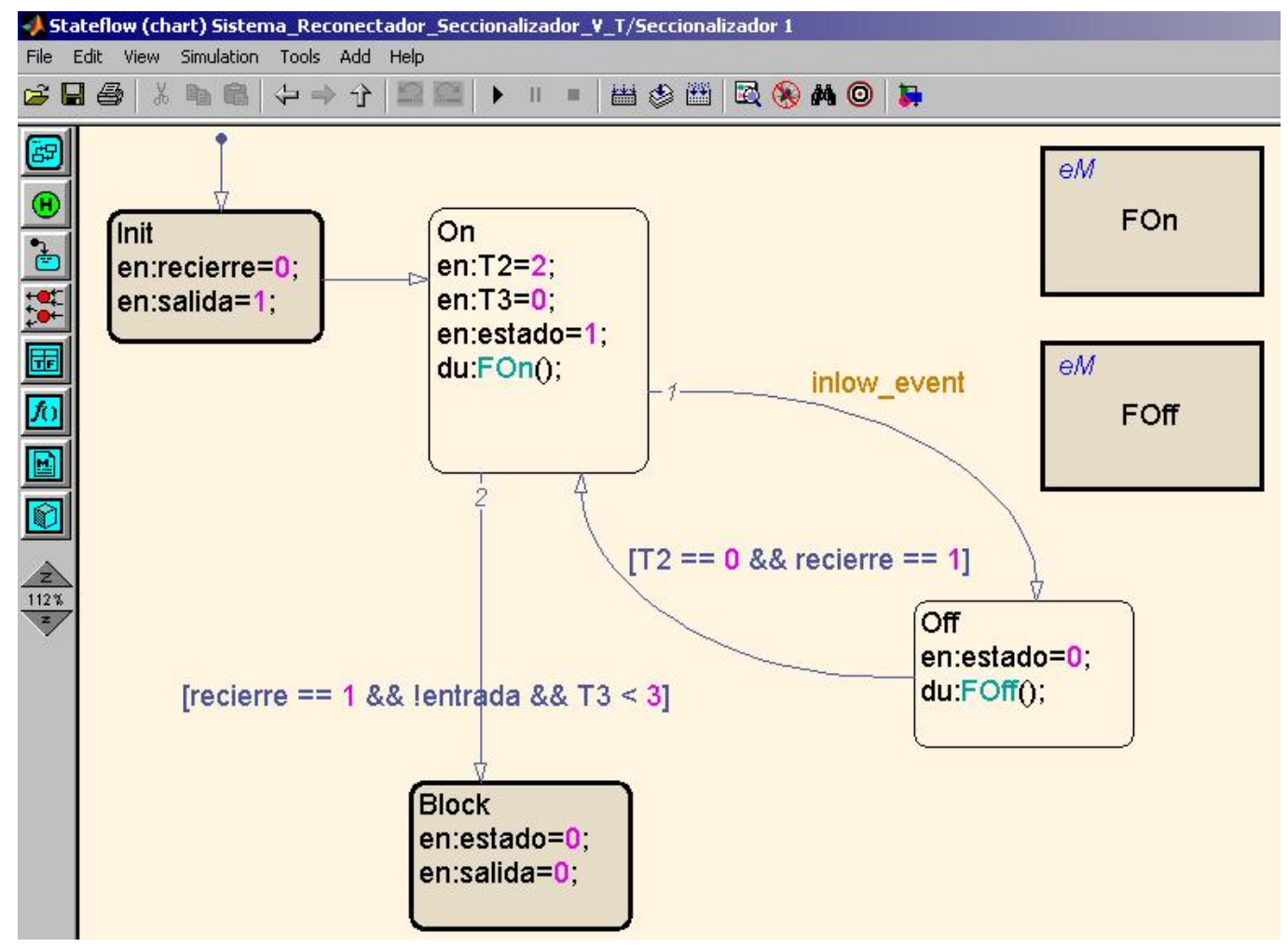

Fig. 6. Máquina de estado finito seccionador.

El re-conectador se encuentra programado con una secuencia $1 A+2 B$, los diagramas temporales deben leerse según sus estados 1 ó 0 de la siguiente manera:

1) Falla: 1 ausencia de falla, 0 presencia de falla.

2) Re-conectador: 1 cerrado, 0 abierto.

3) Seccionador: 1 cerrado, 0 abierto.

\section{RESULTADOS}

A lo largo del presente trabajo se mencionaron dos características que la literatura no tiene correctamente especificadas, la condición de coordinación y la cantidad máxima de seccionadores que pueden ser conectados formando una cadena; para responder estas dos cuestiones se utilizará la Fig. 8 la cual resume los resultados obtenidos de la presente investigación, esta figura rotula en forma detallada la salida por pantalla de la simulación.

\section{Condición de coordinación re-conectador - seccionador tensión - tiempo}

En la Fig. 8 se aprecia que a partir de la falla y tomando como nulo el tiempo de reconexión del reconectador, éste presenta un ciclo rápido caracterizado por la curva rápida (TCC Fast, Time Current-Charateristic Curves) luego del cual abre sus contactos, si se considera que los seccionadores tienen programado un tiempo de apertura (Open Time) nulo, los mismos abren inmediatamente ante la falta de tensión, como consecuencia de ello y luego del tiempo de cierre (Close Time), el re-conectador repone la tensión en el alimentador; el primer seccionador luego de transcurrido su tiempo de cierre (Close Time) tensiona al segundo, el cual con un ciclo similar al primero habilita al tercero luego de su tiempo de cierre (Close Time). 


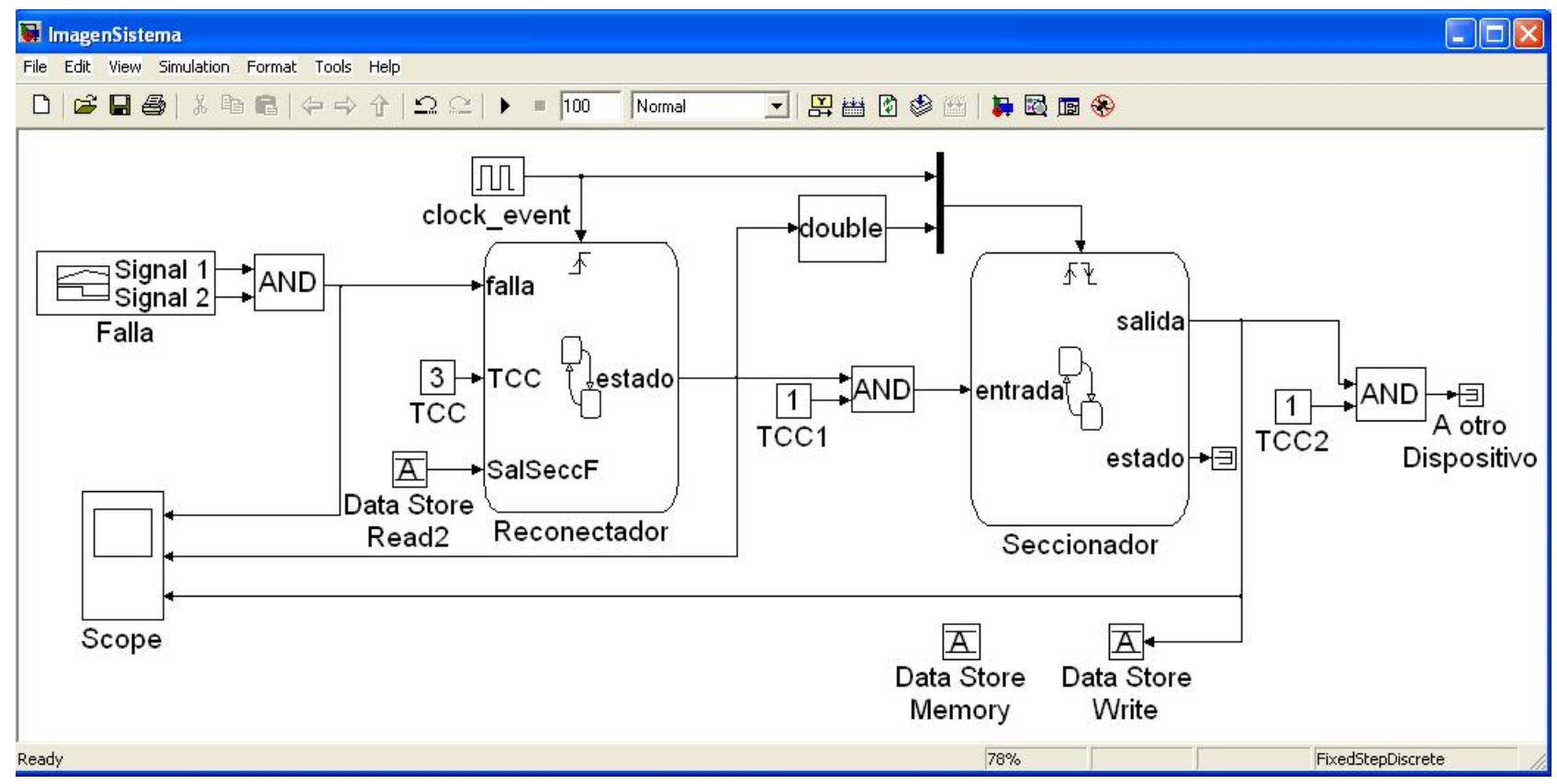

Fig. 7. Diagrama Simulink de la cadena re-conectador / seccionador.

Cuando el tercer seccionador repone el circuito, también repone la falla y el re-conectador reacciona eventualmente con su curva lenta (TCC Low), transcurrida ésta se desconecta y como la curva lenta (TCC Low) del re-conectador es menor que el tiempo de inicialización (Reset Time) del seccionador V-T 3, éste entra en estado de bloqueo despejando la falla, aquí está la clave de la coordinación, el tiempo de inicialización (Reset Time) del seccionador debe ser un poco mayor que el valor del ciclo lento (TCC Low) del re-conectador para la falla del seccionador bajo análisis.

La curva corriente tiempo inverso del re-conectador para la corriente de cortocircuito mínima calculada en la posición del seccionador que cubre la falla, marca el tiempo de sincronización entre los dispositivos.

\section{Límite a la cadena de seccionadores}

De la Fig. 8 es posible obtener la condición que limita el número de seccionadores que pueden integrar la cadena, tiene que ver con el tiempo que el seccionador pueda mantener su prestación faltando la tensión de entrada; esta limitante puede mitigarse con la utilización de baterías recargables en el seccionador.

\section{DISCUSIÓN}

Se aprecia la importancia que adquiere un software que permita un análisis gráfico-dinámico de la coordinación de protecciones en esquemas de protección inteligente, algo que sería bastante complejo de realizar en forma manual. La base de datos tanto de dispositivos como de características merece una especial atención al momento de evaluar un paquete que permita el modelado y simulación de este tipo de sistemas.

Con respecto a la generación distribuida las publicaciones reportan la necesidad de analizar cada caso en forma particular, algunas dan un diagrama de flujo para estudiar ordenadamente las posibles variantes (Martinez et al., 2011). En cuanto a las interferencias que ésta pueda introducir en el esquema de protecciones, ha quedado demostrado en el transcurso del trabajo; la exigencia de un complejo sistema de protecciones propio de la generación distribuida puede aliviar el problema. 


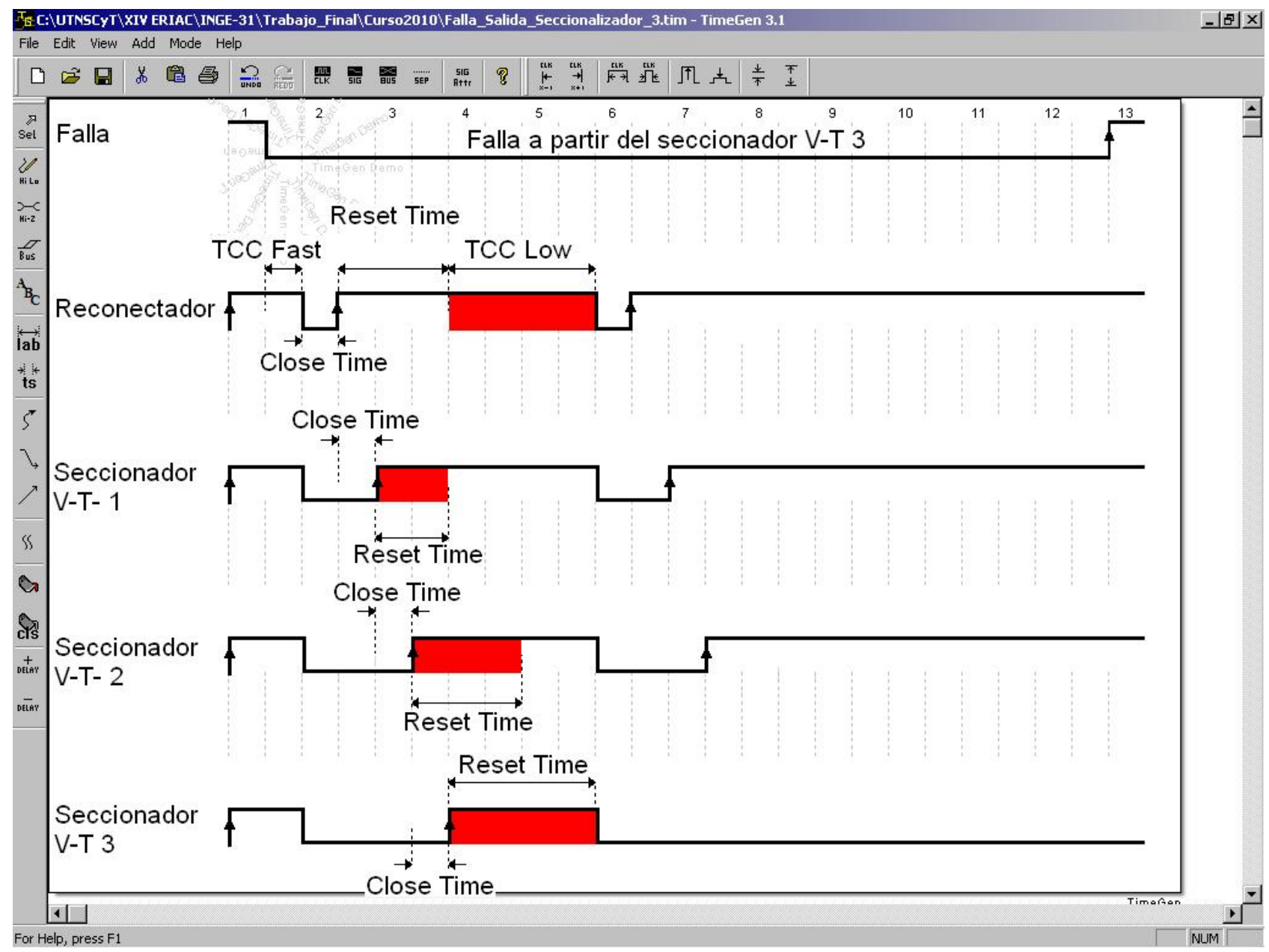

Fig. 8. Diagrama temporal re-conectador - seccionador.

El seccionador tensión - tiempo computarizado, se muestra como una alternativa interesante para la implementación de sistemas de distribución inteligentes y para mitigar problemas de coordinación, si el distribuidor crece en longitud, con el agregado de otro dispositivo se resuelve el problema, situación difícil, sino imposible, si se utilizan fusibles.

Se ha mostrado que el factor que limita la cantidad de seccionadores que integran la cadena, es la capacidad de retención del estado ante la falta de tensión, esto puede disminuirse con fuentes de alimentación propias.

\section{CONCLUSIONES}

De la lectura del presente trabajo se pueden extraer las siguientes conclusiones: 1) El seccionador tensión - tiempo es un dispositivo flexible a la hora de implementar esquemas de distribución inteligente. 2) La coordinación con el re-conectador no depende de los otros dispositivos. 3) La cantidad de seccionadores que se pueden conectar en cadena es teóricamente ilimitada si se utilizan baterías. 4) La implementación de sistemas de distribución inteligentes mejoran los índices de desempeño. 5) Contar con un paquete de modelado y simulación se hace imprescindible a la hora de proyectar y analizar sistemas de distribución inteligente.

\section{REFERENCIAS}

COOPER Power Systems, S270-30-1 Sectionalizers Service Information Installation and Operation Instructions User's Guide, http://www.cooperindustries.com/.../S270-30-1.pdf. Acceso: 9 de Julio (2011). 
Domo J. F., Automatic Distribution Sectionalizing Advances made possible with Micropocessor technology and its role in Distribution Automation, 11 Th. IEEE/PES Transmission and Distribution Conference and Exposition, New Orleans, U.S.A., Apr. 2 - 7 (1989).

Dysko A., Burt G.M., Galloway S., Booth C. and McDonald J.R., UK Distribution System Protection Issues, IET Generation, Transmission and Distribution, 1(4), 679-687 (2007).

Fahey T. S. and Burbure N. V., Three-Phase Reclosers with Single-Phase Tripping Capabality Improve Distribution Systems Reliability, IEEE Power \& Energy Magazine, 6(2), 46-52 (2008).

IEEE C 37.230, IEEE Guide for Protective Relay Application to Distribution Lines, IEEE Power Engineering Society, IEEE Std. C37.230-2007, Feb. 2008.

Kazemi S., Fotuhi-Firuzabad M., Sanaye-Pasand M., and Lehtonen M., Impacts of automatic control systems of loop restoration scheme on the distribution system reliability, IET Generation, Transmission and Distribution, 3(10), 891-902 (2009).

Martinez J. A., de León F., Mehrizi-Sani A., Nehrir M. H., Wang C., and Dinavahi V., Tools for Analysis and Design of Distributed Resources-Part II: Tools for Planning, Analysis and Design of Distribution Networks With Distributed Resources, IEEE Transactions on Power Delivery, 26(3), 1653-1662 (2011).

Sutherland P. E., Goodman F. R. and Short T. A., Feeder and Network Evolution for the Distribution System of the Future, Proceedings of the Transmission and Distribution Conference and Exhibition, 2005/2006 IEEE PES, pp. 348-353, Dallas, U.S.A., May 21-24 (2006).

Walling R. A., Saint R., Dugan R. C., Burke J. and Kojovic L. A., Summary of Distributed Resources Impact on Power Delivery Systems, IEEE Transactions on Power Delivery, 23(3), 16361644 (2008). 